\title{
Estrogen receptor-mediated miR-486-5p regulation of OLFM4 expression in ovarian cancer
}

\author{
Hanyu Ma ${ }^{1, *}$, Tian Tian ${ }^{1, *}$, Shuang Liang ${ }^{1, *}$, Xubin Liu ${ }^{1}$, Hongwei Shen ${ }^{2}$, Meng Xia ${ }^{2}$, \\ Xingyang Liu ${ }^{2}$, Wenhui Zhang ${ }^{1}$, Liantang Wang ${ }^{1}$, Shangwu Chen ${ }^{3}$ and $\mathrm{Li} \mathrm{Yu}^{1}$ \\ ${ }^{1}$ Department of Pathology, The First Affiliated Hospital, Sun Yat-sen (Zhongshan) University, Guangzhou, China \\ 2 Department of Gynecology and Obstetrics, The First Affiliated Hospital, Sun Yat-sen (Zhongshan) University, Guangzhou, \\ China \\ ${ }^{3}$ State Key Laboratory for Biocontrol, Guangdong Key Laboratory of Pharmaceutical Functional Genes, Department of \\ Biochemistry, School of Life Sciences, Sun Yat-sen (Zhongshan) University, Guangzhou, China \\ * These authors have contributed equally to this work \\ Correspondence to: Li Yu, email: yuli5@mail.sysu.edu.cn \\ Shangwu Chen, email: Isschshw@mail.sysu.edu.cn
}

Keywords: OLFM4, ovarian serous adenocarcinoma, estrogen, miR-486-5p

Received: August 19, $2015 \quad$ Accepted: January 27, $2016 \quad$ Published: February 07, 2016

\section{ABSTRACT}

Estrogen signaling influences the development and progression of ovarian tumors, but the underlying mechanisms are not well understood. In a previous study we demonstrated that impairment of estrogen receptor alpha (ERa)mediated olfactomedin 4 (OLFM4) expression promotes the malignant progression of endometrioid adenocarcinoma, and we identified OLFM4 as a potential target of miR-486-5p. In this study we investigated the role of OLFM4 in ovarian serous adenocarcinoma. Ovarian serous adenocarcinoma tissues had reduced OLFM4 expression. Expression of OLFM4 was positively correlated with ERa expression, and estrogen (E2) treatment in ovarian cancer cells induced OLFM4 expression in an ERadependent manner. In contrast to ERa, miR-486-5p levels were inversely correlated with OLFM4 expression in ovarian serous adenocarcinoma. Ovarian cancer cells transfected with miR-486-5p mimics showed decreased OLFM4 mRNA expression, and ovarian cancer cells treated with E2 showed reduced cellular miR-486-5p levels. OLFM4 knockdown enhanced proliferation, migration, and invasion by ovarian cancer cells. Low expression of OLFM4 was also associated with high tumor FIGO stage and poor tumor differentiation. These results suggest OLFM4 is downregulated by miR486-5p, which contributes to ovarian cancer tumorigenesis. Conversely, estrogen receptor signaling downregulates $\mathrm{miR}-486-5 p$ and upregulates OLFM4 expression, slowing the development and progression of ovarian cancer.

\section{INTRODUCTION}

Ovarian cancer, together with endometrial cancer and cervical carcinoma, are the three most common gynecological malignant tumors [1]. Epitheliumderived ovarian serous tumors include benign serous cystadenoma, serous borderline tumors, and malignant serous adenocarcinoma. Ovarian serous cystadenomas are common ovarian lesions that may be precursors of serous borderline tumors, which can in turn progress to ovarian serous adenocarcinomas [2]. Ovarian serous adenocarcinoma accounts for about $75 \%$ of ovarian epithelial tumors and can be highly invasive. The ovaries secrete both estrogen and progesterone, and estrogens play a role in the development, growth, invasion and metastasis of ovarian tumors. Estrogen receptor alpha $(\mathrm{ER} \alpha)$ induces gene expression changes in ovarian cancer cells [3]. High $\mathrm{ER} \alpha$ expression is associated with reduced apoptosis in poorly-differentiated ovarian cancer [4]. However, patients with ER $\alpha$-expression have a better prognosis [5]. The mechanisms of estrogens actions on the development and progression of gynecological tumors is not well understood.

Olfactomedin 4 (OLFM4), also known as hGC-1, 
GW112, hOlf D and pDP4 [6-9], was first cloned from myeloid precursor cells treated with granulocyte colonystimulating factor [7]. OLFM4 is expressed mainly in the gastrointestinal tract, prostate, breast, bone marrow, and pancreas [7, 10]. OLFM4 expression is increased in cancers of the stomach, colon, pancreas, lung, and breast [11-13], and OLFM4 expression is correlated with tumor differentiation and prognosis. Patients with well-differentiated gastric cancer and higher OLFM4 expression have a five-year survival rate higher than patients with poorly differentiated cancer [14]. Induction of OLFM4 regulates adhesion and migration in colon cancer cells [15], promotes $\mathrm{S}$ phase transition and proliferation of pancreatic cancer cells [12], and inhibits migration and invasion of prostate cancer cells [16]. These data suggest that OLFM4 is involved in the pathogenesis and development of various tumors.

We previously demonstrated that aberrant OLFM4 expression also occurs in gynecological tumors. OLFM4 expression has been associated with progression of cervical intraepithelial neoplasia (CIN) and differentiation of cervical cancer [17]. Impairment of ER $\alpha$-mediated OLFM4 expression promotes the malignant progression of endometrioid adenocarcinoma [18]. In a bioinformatics screen for molecules upstream of OLFM4, we discovered one potential regulator, $\mathrm{miR}-486-5 \mathrm{p}$. There is a conserved miR-486-5p-binding site on the 3' UTR of human OLFM4 mRNA. miR-486-5p is enriched in muscle tissue [19], and miR-486-5p targets OLFM4 thereby playing a tumorsuppressive role in gastric cancer [20]. Up-regulation of miR-486-5p also contributes to the progression of pancreatic ductal adenocarcinoma [21]. In the current study, we investigated the expression of OLFM4, ER $\alpha$, and PR in formalin-fixed, paraffin-embedded (FFPE) ovarian tissues, analyzed the expression of miR-486-5p in ovarian tissues and its association with OLFM4 levels and ER $\alpha$ signaling, examined the effects of OLFM4 on ovarian carcinoma cells, and evaluated the role of OLFM4 in the development and progression of ovarian cancer.

\section{RESULTS}

\section{Ovarian serous adenocarcinoma tissues have aberrant olfactomedin 4 expression}

To investigate whether olfactomedin 4 (OLFM4) is associated with ovary tumorigenesis, we examined its expression in normal ovary; serous cystadenoma; serous borderline tumor; and well-, moderately- and poorly-differentiated serous adenocarcinoma using immunohistochemistry (IHC). OLFM4 was stained in the cytoplasm of epithelial cells (Figure 1). The rates of OLFM4 high-expression in these tissues were 16.7, 34.3, $65.1,72.2,50.0$, and $11.9 \%$, respectively (Table S1 and
Table S2). OLFM4 expression in well-differentiated serous adenocarcinoma was higher than normal ovarian epithelium $(P<0.001$, Table S1), indicating an association of OLFM4 expression with ovarian tumorigenesis. Staining intensity of OLFM4 decreased along with the degree of differentiation of serous adenocarcinoma (Figure $1 \mathrm{~N}, 1 \mathrm{R}, 1 \mathrm{~V})$. There was also a difference in OLFM4 expression between well- and poorly-differentiated serous adenocarcinomas $(P<0.001$, Table S2).

\section{Olfactomedin 4 inhibits proliferation, metastasis, and invasion of ovarian serous adenocarcinoma cells}

In a previous study we demonstrated that knockdown of OLFM4 enhances the proliferation, migration, and invasion of endometrial carcinoma cells [18]. We therefore sought to investigate the effects of OLFM4 on ovarian serous adenocarcinoma cells. siRNA knockdown of OLFM4 promoted cell proliferation in both HO8910-pm and SKOV3 cells (Figure 2A), but had no effect on cell apoptosis (Figure 2D). Knockdown of OLFM4 in HO8910-pm cells increased cell migration (Figure 2B) in a scratch wound assay. In a transwell migration assay, the mean number of invaded cells per one visual field (at 200X magnification) was greater with OLFM4 knockdown (mean number $=68$ ) compared with the control group (mean number $=24$ ) (Figure $2 \mathrm{C}$ ). These results suggest that OLFM4 regulates ovarian serous adenocarcinoma cell proliferation and migration. Next, we examined the effects of OLFM4 on cell cycle progression using flow cytometry. HO8910-pm cells with OLFM4 knockdown had decreased numbers of cells in G1 phase and increased numbers in S phase compared with control cells, demonstrating that OLFM4 facilitates cell cycle arrest at G1 (Figure 2E).

\section{OLFM4 expression is associated with the progression and differentiation of ovarian serous adenocarcinoma}

Based on the expression of OLFM4 measured in paraffin-embedded tissues, we analyzed the association of OLFM4 expression with clinicopathological features and prognosis of ovarian serous adenocarcinoma. Low expression of OLFM4 was associated with high tumor FIGO stage and poor tumor differentiation, and not associated with patients' age or metastasis (Table 1). Of the 116 ovarian serous adenocarcinoma tissues, 21 patients died and the cumulative survival rate was $81.9 \%$. The cumulative survival rate was $84.8 \%$ in 46 cases with high expression of OLFM4, and $80.0 \%$ in patients with low-expression of OLFM4. Though this difference is not statistically significant, the survival curve is straighter for patients with high OLFM4 expression (Figure 3). 


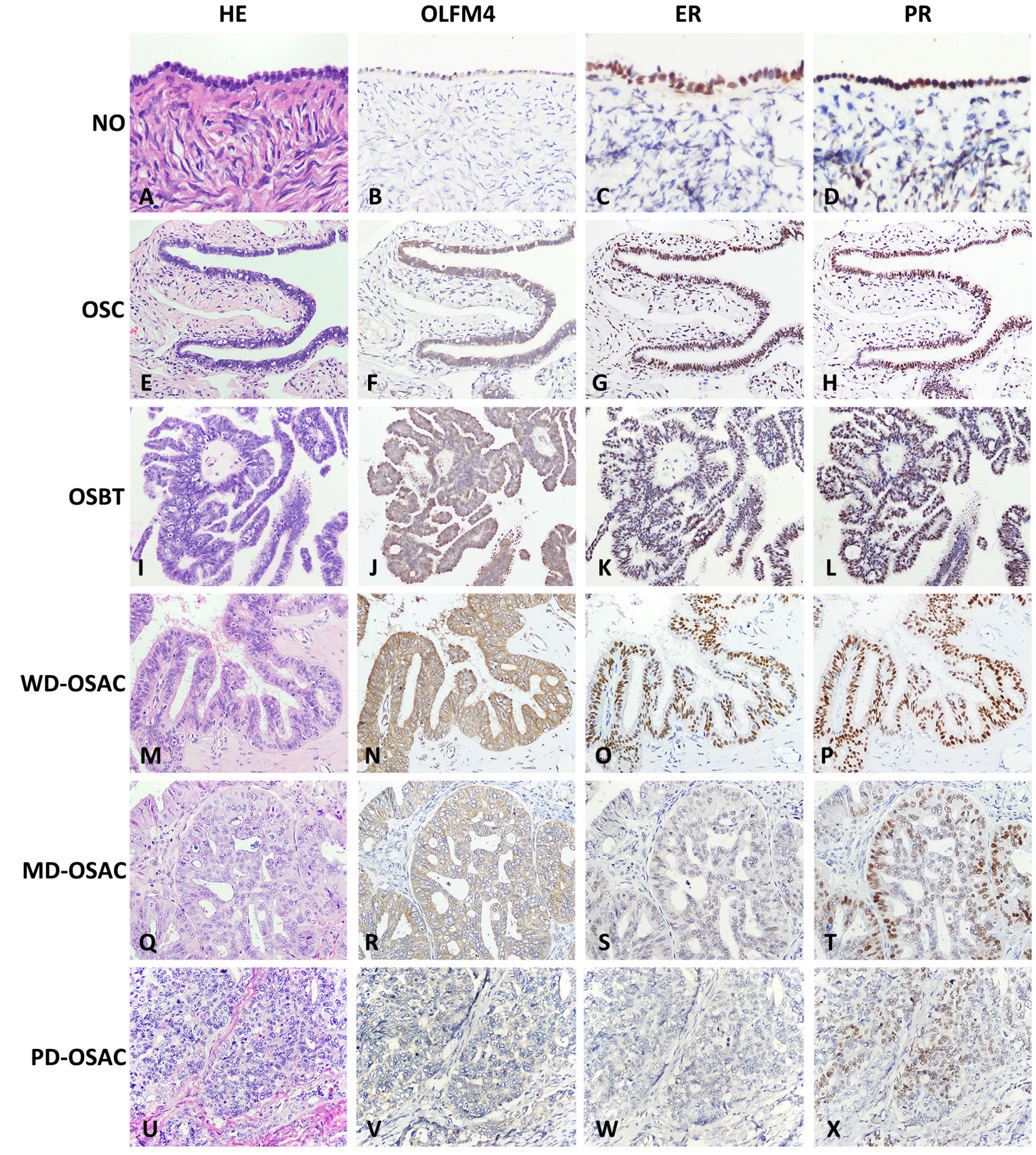

Figure 1: Expression of Olfactomedin 4 (OLFM4), estrogen receptor-a (ER $\alpha)$ and progesterone receptor (PR) in normal ovary (NO); ovarian serous cystadenoma (OSC); ovarian serous borderline tumor (OSBT); well-differentiated ovarian serous adenocarcinoma (WD-OSAC), moderately-differentiated ovarian serous adenocarcinoma (MD-OSAC) and poorly-differentiated ovarian serous adenocarcinoma (PD-OSAC) detected by immunohistochemistry. Haematoxylineosin (HE), Morphology of NO A. OSC E. OSBT I., and WD-OSAC M., MD-OSAC Q. and PD-OSAC (U) stained by haematoxylineosin. OLFM4, OLFM4 staining was undetectable in NO B.. Immunoreactivity of OLFM4 gradually increased from OSC F. OSBT J. to WD-OSAC N., and gradually decreased with lower degrees of differentiation in serous adenocarcinoma (N, R, V). ER, Immunoreactivity of ER gradually decreased from NO C. OSC G. OSBT K. to WD-OSAC O. ER staining was hardly detectable in MD-OSAC S. and PDOSAC W. PR, Immunoreactivity of PR gradually decreased from NO D. OSC H. OSBT L., WD-OSAC P., MD-OSAC T. to PD-OSAC X. 
Table 1: Association of OLFM4 expression detected by IHC with clinicopathological features of ovarian serous adenocarcinoma

\begin{tabular}{|c|c|c|c|}
\hline \multirow[b]{2}{*}{ Parameters } & \multicolumn{2}{|c|}{ OLFM4 expression } & \multirow[b]{2}{*}{$\boldsymbol{P}$} \\
\hline & High & Low & \\
\hline \multicolumn{4}{|l|}{ Age } \\
\hline$>50(n=54)$ & 21 & 33 & 0.875 \\
\hline$\leqq 50(n=62)$ & 25 & 37 & \\
\hline \multicolumn{4}{|l|}{ FIGO stages } \\
\hline I-II $(n=44)$ & 23 & 21 & 0.030 \\
\hline III-IV $(n=72)$ & 23 & 49 & \\
\hline \multicolumn{4}{|l|}{ Histological grade } \\
\hline Well $(n=18)$ & 13 & 5 & $<0.371$ \\
\hline Moderate $(n=56)$ & 28 & 28 & \\
\hline Poor $(n=42)$ & 5 & 37 & \\
\hline \multicolumn{4}{|l|}{ Metastasis } \\
\hline No $(n=23)$ & 11 & 12 & 0.371 \\
\hline Yes $(n=93)$ & 35 & 58 & \\
\hline
\end{tabular}

High: High-expression; Low: Low-expression.

Table 2: Co-relationship of OLFM4 level with ER $\alpha$ and PR expression in ovarian tissues based on IHC data

\begin{tabular}{|c|c|c|c|c|c|c|c|c|}
\hline \multirow{2}{*}{$\begin{array}{l}\text { OLFM } \\
4\end{array}$} & \multicolumn{2}{|c|}{ NO $(n=18)$} & \multicolumn{2}{|c|}{$\operatorname{OSC}(n=35)$} & \multicolumn{2}{|c|}{ OSBT $(n=43)$} & \multicolumn{2}{|c|}{ OSAC $(n=116)$} \\
\hline & High & Low & High & Low & High & Low & High & Low \\
\hline \multicolumn{9}{|l|}{ ER $\alpha$} \\
\hline High & 3 & 0 & 6 & 6 & 9 & 19 & 13 & 33 \\
\hline Low & 8 & 7 & 10 & 13 & 6 & 9 & 6 & 64 \\
\hline$P$ & \multicolumn{2}{|c|}{0.130} & \multicolumn{2}{|c|}{0.713} & \multicolumn{2}{|c|}{0.606} & \multicolumn{2}{|c|}{0.005} \\
\hline $\mathrm{r}$ & \multicolumn{2}{|c|}{ - } & \multirow{2}{*}{\multicolumn{2}{|c|}{-}} & \multirow{2}{*}{\multicolumn{2}{|c|}{-}} & \multirow{2}{*}{\multicolumn{2}{|c|}{0.260}} \\
\hline \multicolumn{3}{|l|}{ PR } & & & & & & \\
\hline High & 2 & 1 & 8 & 4 & 17 & 11 & 8 & 38 \\
\hline Low & 11 & 4 & 13 & 10 & 11 & 4 & 6 & 64 \\
\hline$P$ & \multicolumn{2}{|c|}{0.814} & \multicolumn{2}{|c|}{0.561} & \multicolumn{2}{|c|}{0.408} & \multicolumn{2}{|c|}{0.154} \\
\hline $\mathrm{r}$ & \multicolumn{2}{|c|}{ - } & \multicolumn{2}{|c|}{ - } & \multicolumn{2}{|c|}{ - } & \multicolumn{2}{|r|}{-} \\
\hline
\end{tabular}

NO: normal ovary; OSC: ovarian serous cystadenoma; OSBT: ovarian serous borderline tumor; OSAC: ovarian serous adenocarcinoma. High: high-expression; Low: low-expression.

\section{OLFM4 expression is regulated by ERa signaling}

Estrogen receptor signaling regulates the expression of OLFM4 in endometrioid adenocarcinoma [18]. To investigate whether this regulation also occurs in ovarian serous adenocarcinoma, we examined the expression of ER $\alpha$ and PR in ovarian tissues using IHC (Figure 1). The rates of ER $\alpha$ high-expression in normal ovary, serous cystadenoma, serous borderline tumor, and ovarian serous adenocarcinoma were 61.1, 45.7, 34.9 and $16.4 \%$, respectively (Table S3). High-expression rates of PR in these tissues were 72.2, 60.0, 65.1 and $12.1 \%$, respectively (Table S3). Expression of OLFM4 in ovarian serous adenocarcinoma was positively correlated with the expression of ER $\alpha$ but not PR (Table 2).

Next, we used ovarian cancer cells, SKOV3 and HO8910-pm, to investigate the association of OLFM4 expression with estrogen receptor signaling. Both
SKOV3 and HO8910-pm cells expressed OLFM4, ER $\alpha$, and PR. OLFM4 and ER $\alpha$ expression in SKOV3 cells, a moderately-differentiated ovarian serous adenocarcinoma cell line, was higher than the expression in HO8910-pm, a poorly-differentiated ovarian serous adenocarcinoma cell line (Figure 4), further suggesting the correlation of OLFM4 expression with ER $\alpha$ expression and degree of tumor differentiation. Stimulation of cells with $17 \beta$-estradiol (E2) increased the production of OLFM4 mRNA and protein in HO8910-pm cells, while an estrogen receptor antagonist, ICI 182780 , attenuated the OLFM4 mRNA increase induced by E2 (Figure 5A). Knockdown of ER $\alpha$ reduced the E2-induced expression of OLFM4 mRNA in HO8910-pm cells (Figure 5B, 5C). These results suggest that $E R \alpha$-mediated signaling enhances expression of OLFM4. Thus, estrogen receptor signaling in the development of ovary serous adenocarcinoma may be partially due to the regulation of OLFM4. 
Table 3: Co-relationship of OLFM4 level with miR-486-5p expression

\begin{tabular}{|c|c|c|c|c|c|c|}
\hline \multirow{2}{*}{ OLFM4 } & \multicolumn{2}{|c|}{ OSC $(n=35)$} & \multicolumn{2}{|c|}{ OSBT $(n=43)$} & \multicolumn{2}{|c|}{ OSAC $(n=116)$} \\
\hline & High & Low & High & Low & High & Low \\
\hline \multicolumn{7}{|l|}{ miR-486-5p } \\
\hline High & 5 & 7 & 10 & 18 & 7 & 39 \\
\hline Low & 10 & 13 & 4 & 11 & 23 & 47 \\
\hline$P$ & \multicolumn{2}{|c|}{0.713} & \multicolumn{2}{|c|}{0.606} & \multicolumn{2}{|c|}{0.034} \\
\hline $\mathrm{r}$ & \multicolumn{2}{|c|}{-} & \multicolumn{2}{|c|}{-} & \multicolumn{2}{|c|}{-0.197} \\
\hline
\end{tabular}

OSC: ovarian serous cystadenoma; OSBT: ovarian serous borderline tumor;

OSAC: ovarian serous adenocarcinoma. High: high-expression; Low: low-expression.

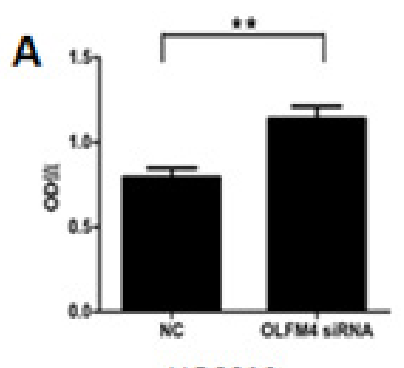

HO8910-pm

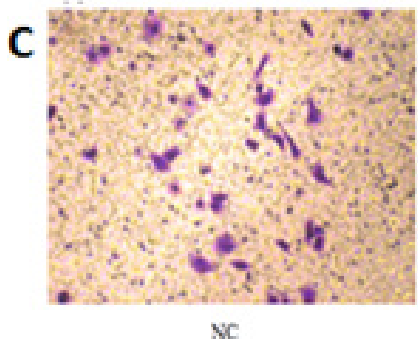

$\mathrm{NC}$
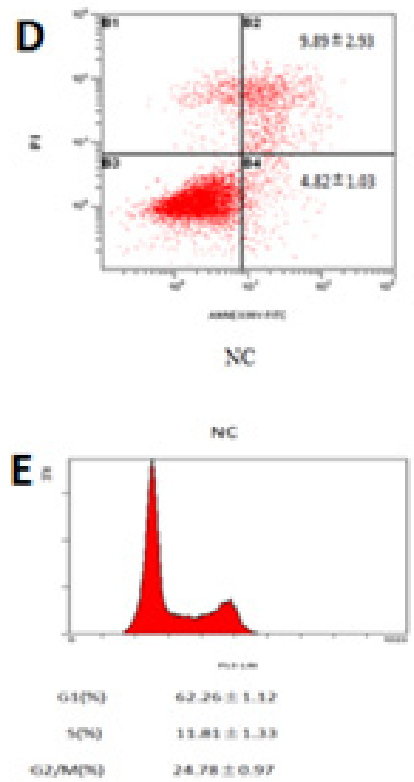
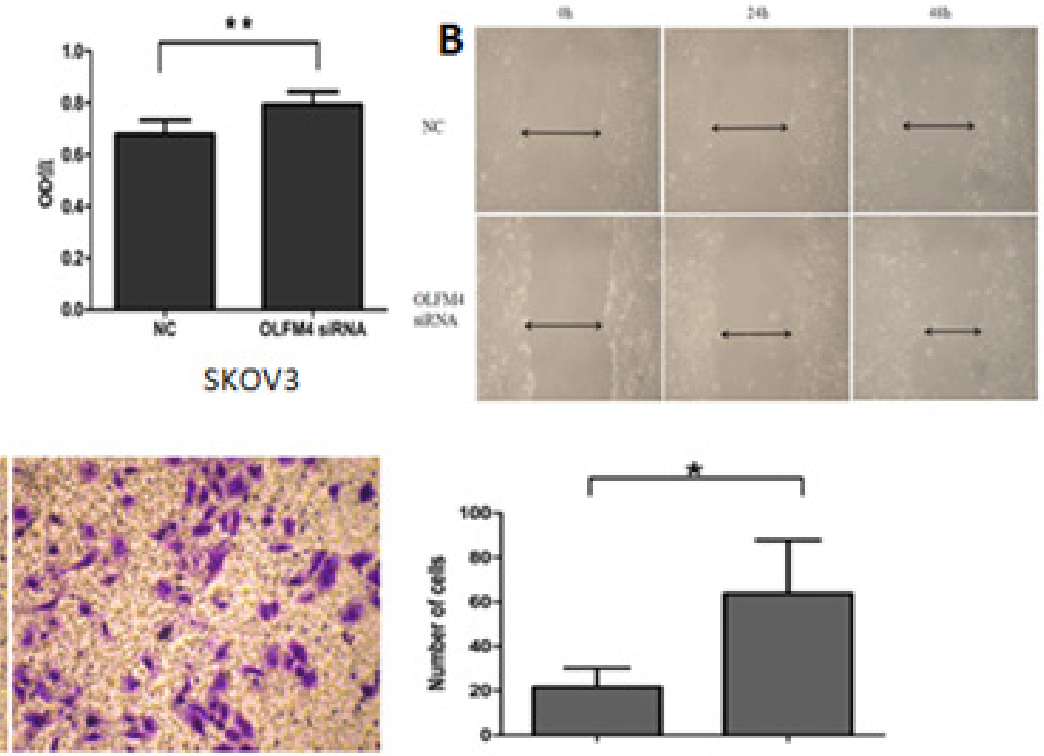

OCFM4 siseA

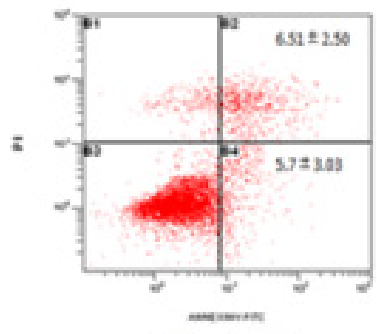

OLFM4 SiRNA

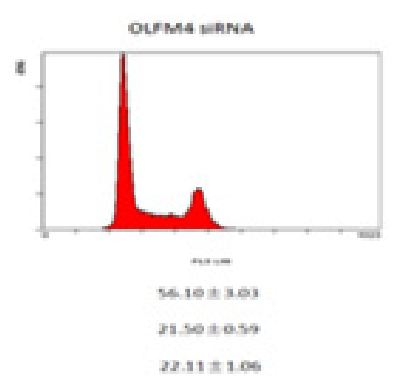

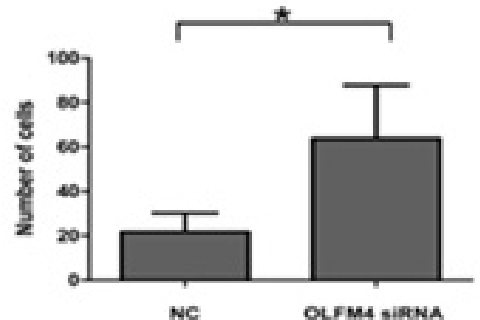

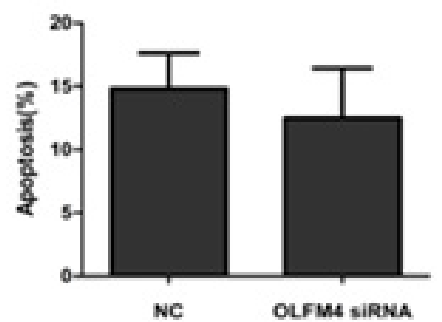

$\square$ Negative ceotrol aikVA oumu siks

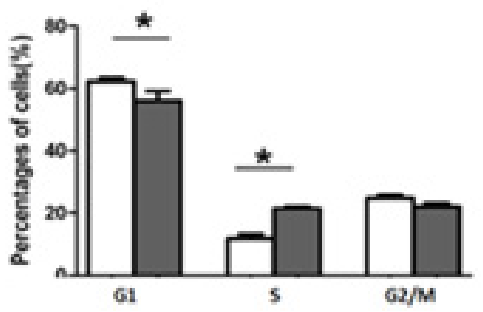

Figure 2: Effects of OLFM4 on ovarian serous adenocarcinoma cells. A. Effects of OLFM4 on proliferation of HO8910-pm and SKOV3 measured by MTT $(* * P<0.01)$. B. OLFM4 knockdown promoted migration of HO8910-pm cells by scratch wound assay. C. OLFM4 knockdown promoted invasion of HO8910-pm cells $\left({ }^{*} P<0.05\right)$ by transwell invasion assay. D. Treatment of HO8910-pm cells with OLFM4 siRNA had no effect on cell apoptosis. E. Knockdown of OLFM4 in HO8910-pm cells transfected with specific siRNA resulted in decrease in G1 phase cells and increase in $\mathrm{S}$ phase cells $(* P<0.05)$. 


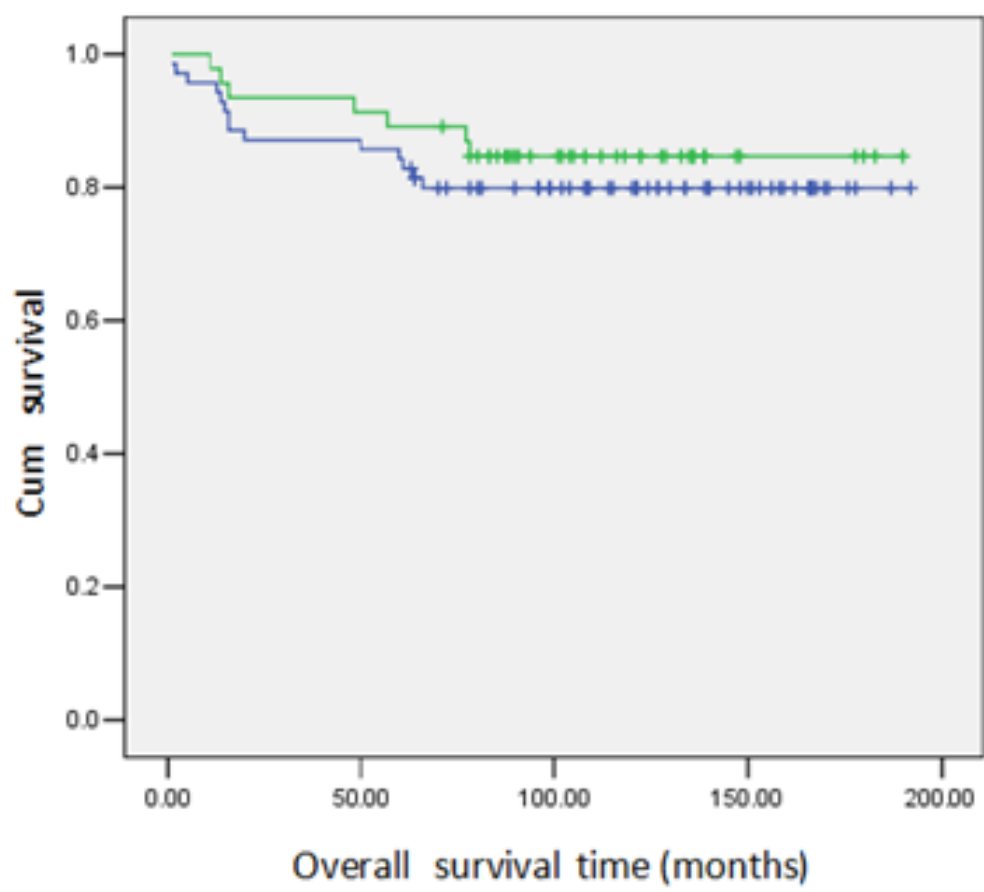

OLFM4

$\Pi$ Low-expression

$\Pi$ high-expression

+ Low-expression-censored

+ high-expression-censored

Overall survival time (months)

Figure 3: Cumulative survival curve of patients with ovarian serous adenocarcinoma and expression of OLFM4.

A

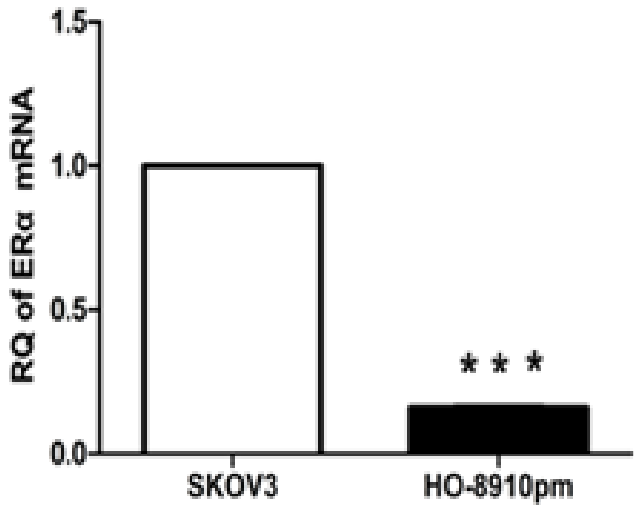

C

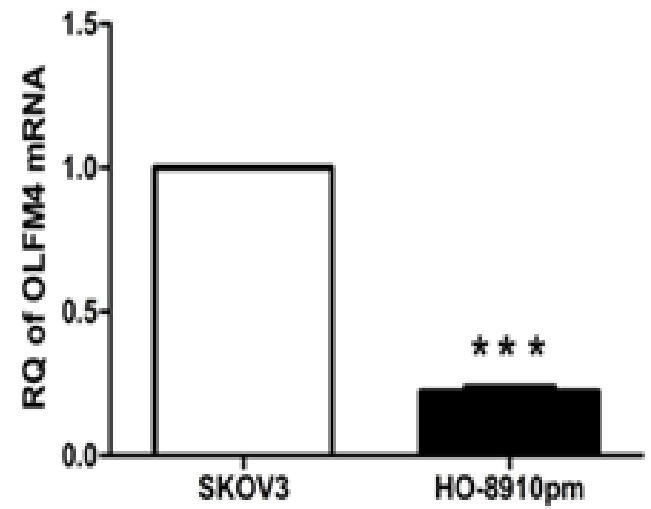

B

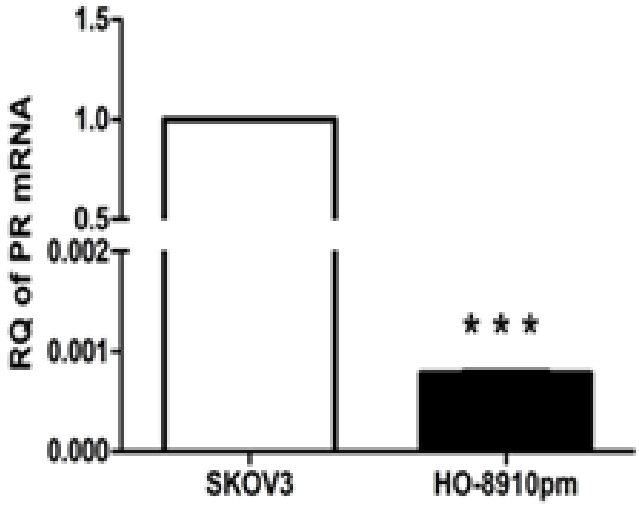

D

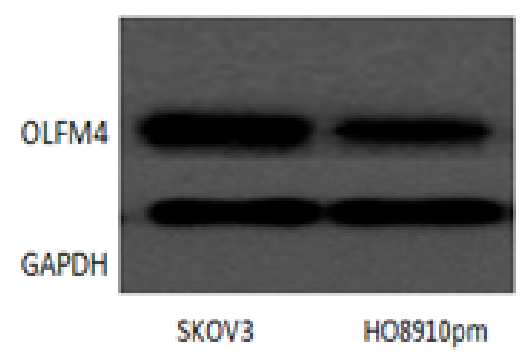

Figure 4: Expression of ERa A. PR B. OLFM4 C. mRNAs, and OLFM4 protein D. in ovarian serous carcinoma cells, $* * * P<0.001$. 


\section{$E R \alpha-m e d i a t e d ~ m i R-486-5 p$ regulates expression of OLFM4}

Bioinformatics analysis identified a putative miR486-5p target site on the 3' UTR sequence of human OLFM4 [20]. miR-486-5p levels were measured in FFPE tissues of normal ovary, ovarian serous cystadenoma, ovarian serous borderline tumor, and ovarian serous adenocarcinoma by real-time PCR. There was a difference in miR-486-5p levels between normal ovary and ovarian serous adenocarcinoma (Figure 6A). To compare with the OLFM4 expression data measured by IHC, the average value of miR-486-5p in normal tissues was calculated and all other samples were then calibrated to normal tissues. A relative value above the average level in normal tissues was assigned as miR-486-5p high expression, and viceversa. Rates of miR-486-5p high expression in ovarian serous cystadenoma, ovarian serous borderline tumor, and ovarian serous adenocarcinoma tissues were 42.9, 32.6 and $25.9 \%$, respectively (Table S4). miR-486-5p expression was decreased in ovarian serous adenocarcinoma as compared to normal ovary, and inversely correlated with OLFM4 expression in ovarian serous adenocarcinoma
(Table 3).

When OLFM4 expression was analyzed by Western blot and miR-486-5p expression was measured by real-time PCR in frozen fresh human ovarian cancer and normal tissues, we found that miR-486-5p levels in ovarian cancer tissues were lower than in normal tissues (Figure 6B) and that OLFM4 was more frequently detectable in ovarian cancer tissues as compared with normal tissues (Figure 6C).

To investigate whether OLFM4 expression is regulated by miR-486-5p, miR-486-5p was overexpressed in adenocarcinoma cells. HO8910-pm and SKOV3 cells transfected with miR-486-5p mimics, had decreased OLFM4 mRNA levels, indicating that OLFM4 is a potential target of miR-486-5p in ovarian serous adenocarcinoma (Figure 6D, 6E). Interestingly, when ovarian cancer cells were treated with E2, miR-486-5p expression was reduced (Figure 6F). The estrogen receptor antagonist ICI 182780 or knockdown of ER $\alpha$ attenuated E2-induced changes in miR-486-5p expression in SKOV3 cells (Figure 6F), supporting a potential inhibitory effect of $E R \alpha$ signaling on miR-486-5p expression.

A

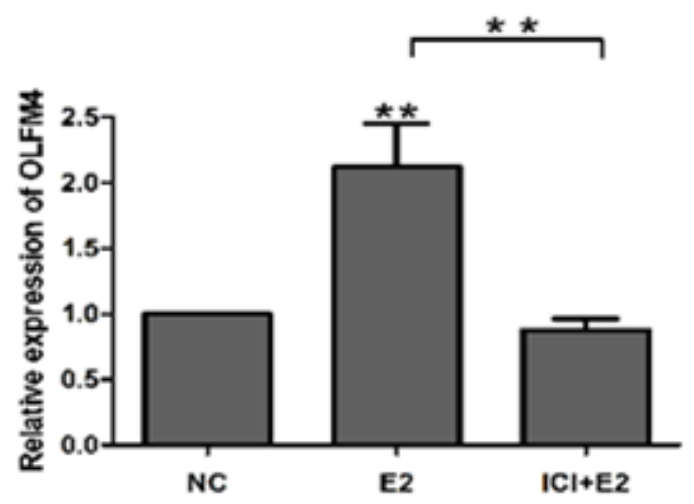

B

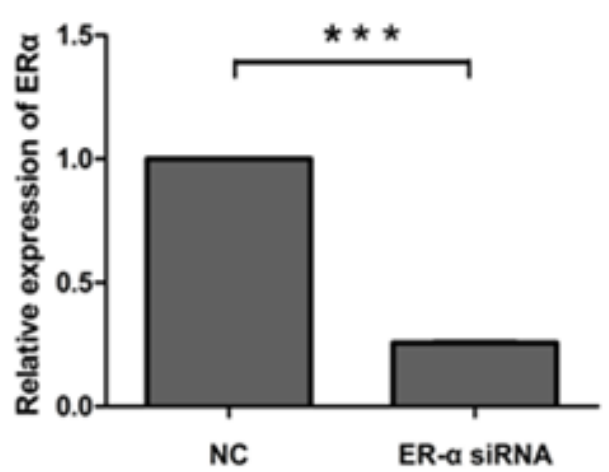

C

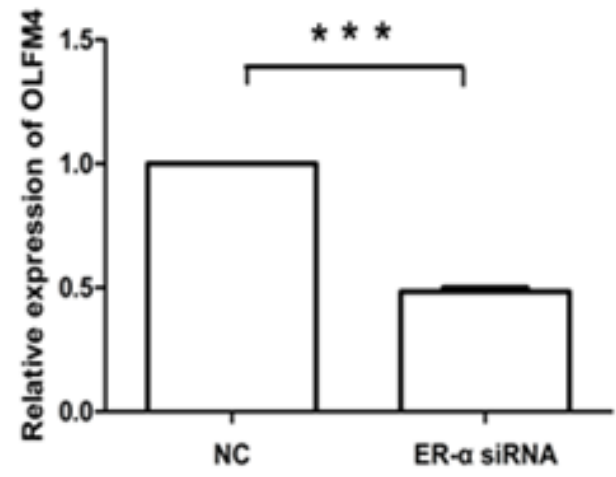

Figure 5: ERo-mediated regulation of OLFM4 expression in HO8910-pm cells. A. E2 induced the expression of OLFM4 and estrogen receptor antagonist ICI 182780 attenuated the OLFM4 mRNA increase induced by E2. B. Knockdown of ER $\alpha$ expression with siRNA. C. Down-regulation of ER $\alpha$ with siRNA reduced the E2-induced expression of $O L F M 4$. *** $P<0.001, * * P<0.01$. NC: negative control. 
A

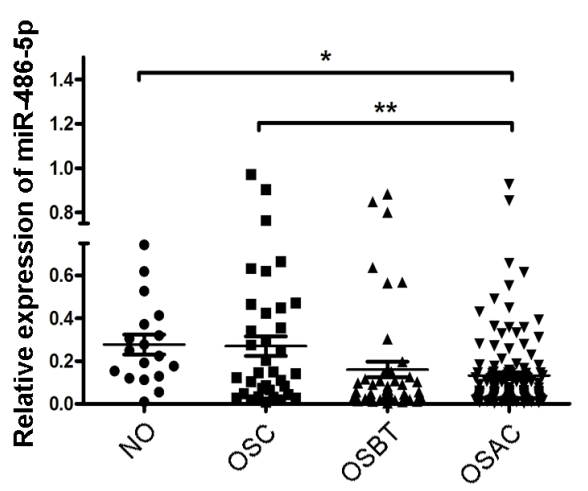

B

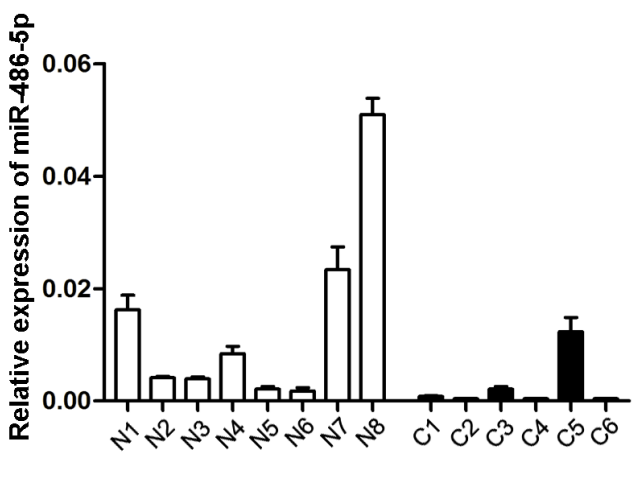

C

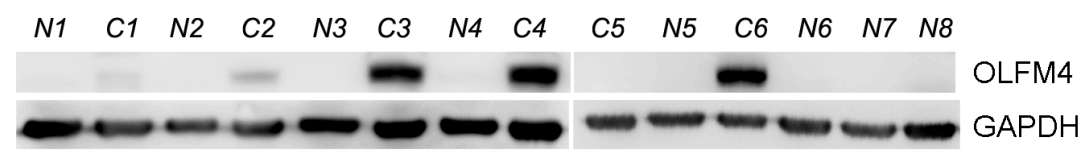

D

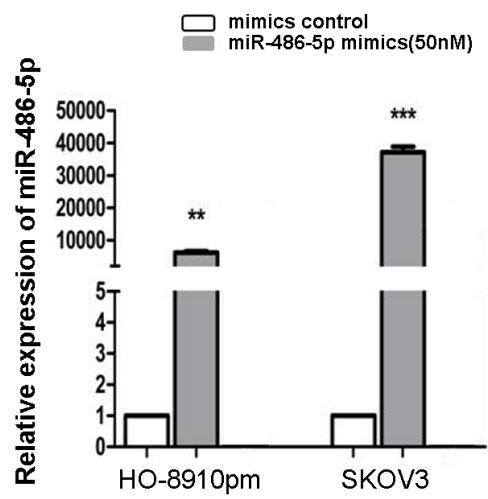

$\mathbf{F}$

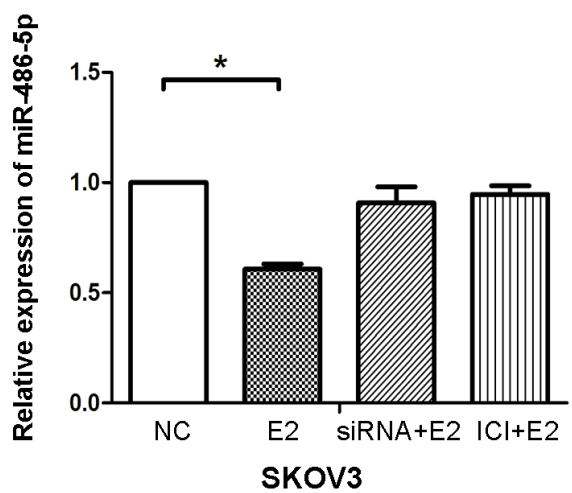

E

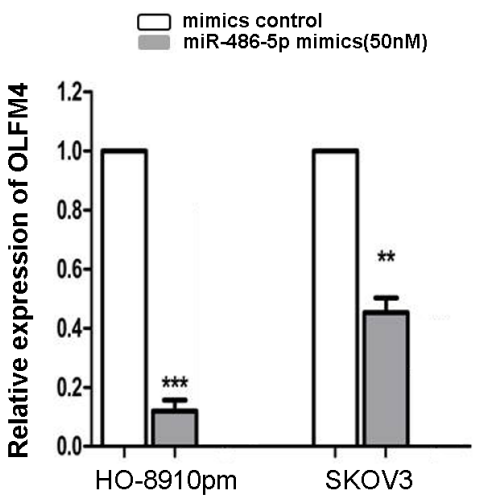

G

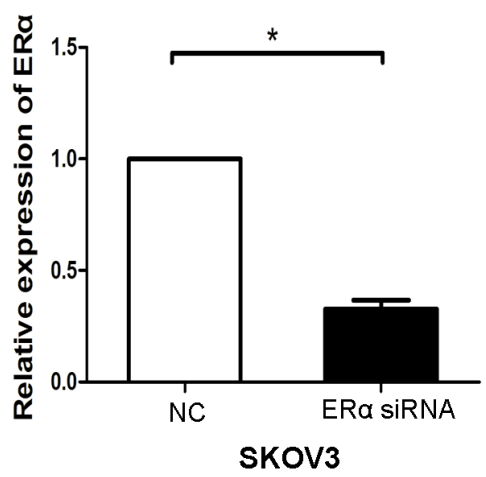

Figure 6: Estrogen regulates the expression of miR-486-5p, which targets OLFM4. A. Comparison of miR-486-5p levels among formalin-fixed, paraffin-embedded (FFPE) tissues of normal ovary (NO), ovarian serous cystadenoma (OSC), ovarian serous borderline tumor (OSBT), and ovarian serous adenocarcinoma (OSAC) detected by IHC. B. miR-486-5p expression in frozen fresh ovarian serous adenocarcinoma and normal tissues detected by real-time PCR. C and N represent individual tissues of serous adenocarcinoma and normal ovary, respectively. C. OLFM4 expression in frozen fresh ovarian serous adenocarcinoma and normal tissues was detected by Western blot. D. Transfection of miR-486-5p mimics increased miR-486-5p levels in ovarian cancer cells. E. Increased miR-486-5p led to reduced OLFM4 mRNA levels. F. Treatment with estrogen (E2) resulted in decreased miR-486-5p levels. Treatment with estrogen receptor antagonist ICI 182780 (ICI) or knockdown of ER $\alpha$ with ER $\alpha$-specific siRNA attenuated E2-induced decrease of miR-486-5p levels in SKOV3 cells. G. Knockdown of ER $\alpha$ expression with ER $\alpha$-specific siRNA in SKOV3 cells. NC: negative control, $* * * P<0.001, * * P<$ 0.01 , and $* P<0.05$. 


\section{DISCUSSION}

Aberrant expression of OLFM4 has been observed in some cancerous tissues, especially in those of the digestive system. In our previous studies we demonstrated that OLFM4 is also functionally associated with gynecological tumors such as cervical cancer and endometrial adenocarcinoma $[17,18]$. In the current study, aberrant OLFM4 expression was observed in ovarian serous adenocarcinoma tissues. Mechanistic studies demonstrated estrogen receptor-mediated miR-486-5p targeting of OLFM4 in ovarian cancer

Scratch wound and transwell migration assays indicated that OLFM4 inhibits cell migration and invasion of ovarian cancer cells (Figure 2B, 2C). However, expression of OLFM4 was not associated with metastasis in our clinicopathological analysis (Table 1). This discrepancy is probably due to small sample sizes. There were 58 patients with low OLFM4 expression in a total 93 metastasis patients, but only 35 patients with high OLFM4 expression. Although an association was not observed, there was a tendency for patients with low OLFM4 expression to be prone to metastasis.

Both up- and down-regulation of miR-486 have been associated with the development and progression of tumors. Down-regulation of miR-486 contributes to the progression and metastasis of breast, liver and lung cancers [22-24] and up-regulation of miR-486 promotes the progression of pancreatic ductal adenocarcinoma [21] and gliomas [25]. miR-486 targets Pim-1 kinase in lung cancer [26], OLFM4 in gastric cancer [20], CLDN10 and CITRON in hepatocellular carcinoma [24], and plasminogen activator inhibitor-1 in human myxoid liposarcoma [27]. Although miR-486 is involved in many tumors and targets different effecter molecules, our results support the idea that OLFM4 is one of potential miR-486 targets.

Expression of ER $\alpha$ is associated with estrogendependent growth, invasion, and response to endocrine therapy in ER $\alpha$-positive ovarian cancer, but the exact mechanism remains less clear. Low concentrations of estrogen do not affect the proliferation of ovarian cancer cells. In contrast, high concentrations of estrogen inhibits survival rate of ovarian cancer cells [28]. A combination of estrogen and progesterone decreases cell proliferation and inhibits the expression of Bcl-2 via let-7a and miR$34 \mathrm{~b}$ in ovarian cancer cells [28]. miR-206 inhibits cellular proliferation and invasion of ER $\alpha$-positive ovarian cancer cells [29]. The phosphoinositide 3-kinase (PI3K)/AKT pathway is involved in estrogen-induced metastasis in ovarian cancer cells [30]. OLFM4 is regulated by estrogen in the human endometrium [31]. Here we demonstrated that ER $\alpha$-mediated signaling regulates the expression of miR-486-5p, which targets OLFM4 in ovarian cancer. Our study suggests that the role of ER signaling in ovarian cancer may be partially due to the regulation of mi-486-
$5 \mathrm{p}$ and $O L F M 4$.

\section{MATERIALS AND METHODS}

\section{Subjects and tissue samples}

Formalin-fixed, paraffin-embedded (FFPE) tissues of ovarian serous adenocarcinoma $(n=116$; mean patient age $=50$ ) and corresponding patient clinicopathological data were collected from December 1998 to December 2008 at the Department of Pathology, First Affiliated Hospital of Sun Yet-sen University. Patients were followed up until 1 June 2014 or until death. All specimens were obtained from surgery. None had received preoperative radiotherapy, chemotherapy or hormone drug therapy. Patients were evaluated in accordance with the International Federation of Gynecology and Obstetrics (FIGO) criteria for staging ovarian cancers. The number of cases classified as FIGO stage I, II, III and IV were 22, 22, 59 and 13, respectively. Histological grade was determined in accordance with the World Health Organization (WHO) grading system. The number of cases classified as WHO grade 1, 2 and 3 were 18, 56 and 42, respectively. FFPE specimens of normal ovary $(n=18)$, ovarian serous cystadenoma $(n=35)$, and ovarian serous borderline tumor $(n=43)$ were also collected. Frozen fresh tissues of well-differentiated ovarian serous adenocarcinoma $(n=6)$ and normal ovary $(n=8)$ were collected.

\section{Steroid hormones and chemicals}

$17 \beta$-estradiol (E2) was purchased from SigmaAldrich (St. Louis, MO, USA). ICI 182780 was purchased from Tocris Cookson Ltd (Bristol, UK).

\section{Immunohistochemistry}

Immunohistochemistry (IHC) was conducted as described previously with antibodies to OLFM4 (Lifespan, rabbit anti-human polyclonal, diluted at 1/100; Seattle, USA), ER $\alpha$ (Kit-0012-2, rabbit anti-human monoclonal, ready to use; Maixin Biotechnology, Fuzhou, Fujian, China), PR (Kit-0013-2, rabbit anti-human monoclonal, ready to use; Maixin Biotechnology), and horseradish peroxidase-labeled secondary antibody (Maixin Biotechnology) in accordance with manufacturer's instructions. Color was developed with diaminobenzidine (Dako Corp, Carpinteria, CA, USA) incubated for 5-10 min at room temperature. Slides were counterstained with haematoxylin and examined by light microscopy.

Staining intensity was graded according to the following criteria described in our previous study [18]: 0 (no staining), 1 (weak staining, light yellow), 2 (moderate 
staining, yellow with brown), and 3 (strong staining, brown). The percent staining was graded according to the proportion of positive stained cells as follows: 0 for $\leq 5 \%$ positive cells; 1 for $6-25 \%$ positive cells; 2 for 26 $50 \%$ positive cells and 3 for $\geq 51 \%$ positive cells. The immunoreactive score (IRS) was used to evaluate results. IRS $=$ staining intensity $\times$ percent of positive cells. An IRS score of 4 and higher was regarded as high expression.

\section{Cell culture}

SKOV3, a moderately-differentiated ovarian serous adenocarcinoma cell line, was purchased from Shanghai Institute for Biological Sciences (Shanghai, China). HO8910-pm, a poorly-differentiated ovarian serous adenocarcinoma cell line, was reserved in Department of Gynecology, the First Affiliated Hospital, Sun Yat-sen University. The cells were cultured in DMEM (Hyclone Laboratories) supplemented with $10 \%$ fetal bovine serum (GIBCO, Australia), $100 \mathrm{U} / \mathrm{ml}$ penicillin $\mathrm{G}$ and $100 \mu \mathrm{g} /$ $\mathrm{ml}$ streptomycin in a humidified atmosphere of $5 \% \mathrm{CO}_{2}$, at $37^{\circ} \mathrm{C}$.

\section{Cell transfection}

SKOV3 and HO8910-pm cells were seeded in 6-well plates for $24 \mathrm{hrs}$ before transfection. Cells grown to $30-50 \%$ confluence were transfected using lipofectamine RNAiMAX (Invitrogen) with one of small interfering RNA (siRNA) duplexes specific for human $\operatorname{ER} \alpha$ and OLFM4, and miR-486-5p mimics or corresponding negative control siRNA or negative control mimics according to the manufacturer's protocol. The specificity and efficacy of siRNA or miRNA were initially evaluated.

\section{Cell proliferation assay}

Cell proliferation was estimated using 3-(4,5-dimethylthiazol-2-yl)-2,5-diphenyltetrazolium bromide (MTT; Sigma-Aldrich). $9 \times 10^{3}$ cells per well were seeded in 96-well plates. Cells grown to $30-50 \%$ confluence were transfected with siRNA specific for OLFM4 or negative control siRNA for $72 \mathrm{hrs}$. At the end of the treatment, $10 \mathrm{ul}$ of MTT solution $(5 \mathrm{mg} / \mathrm{ml}$ in phosphate-buffered saline) was added to the medium and the cells were incubated at $37^{\circ} \mathrm{C}$ for $4 \mathrm{hrs}$. The MTTcontaining medium was removed, and the cells were lysed with 200ul dimethyl sulfoxide for $20 \mathrm{~min}$. Absorbance values were measured using a microplate reader at 570 $\mathrm{nm}$, and the results were plotted as mean $\pm \mathrm{SD}$.

\section{Cell apoptosis assay}

$3 \times 10^{5}$ cells were pelleted by centrifugation, washed twice with cold PBS, and resuspended in $500 \mu$ cold Annexin binding buffer containing $5 \mu$ Annexin V-FITC and $5 \mu$ propidium iodide. The cells were incubated for 15 minutes in the dark at room temperature and analyzed using a Becton Dickinson FACScan (Becton Dickinson Immunocytometry Systems, San Jose, CA). The experiment was performed in triplicate, independently.

\section{Cell cycle analysis}

$3 \times 10^{5}$ cells were washed twice with cold PBS and fixed with $70 \%$ ethanol overnight. Cells were washed with PBS, pelleted by centrifugation, and resuspended in $100 \mathrm{ul} \mathrm{RNase} A(100 \mathrm{ug} / \mathrm{ml})$ and incubated at $37^{\circ} \mathrm{C}$ for 30 min. With an addition of 400 ul propidium iodide, cells were then incubated for 15 minutes in the dark at room temperature and analyzed with flow cytometry.

\section{Scratch wound assay}

Cells were plated in 6-well plates and incubated overnight until $30 \%-50 \%$ confluent, then transfected with 100 nM OLFM4 siRNA or negative control siRNA. Confluent cells wounded by scratching with a $20 \mathrm{ul}$ micropipette tip. Wells were washed three times with PBS to remove dislodged cells. 'Wound closure' was monitored at $0,24,48 \mathrm{hrs}$ and photographed through an inverted microscope.

\section{Transwell migration and invasion assays}

$4 \times 10^{4}$ cells were plated in serum-free media in the upper chamber of 24-well Transwell Chambers (Corning Incorporated, Life Sciences), while media containing 10\% FBS were added to the lower chamber as chemoattractant. The cells were incubated at $37^{\circ} \mathrm{C}$ in a 24 -well plate and allowed to invade through the matrigel (BD Biosciences) for $36 \mathrm{hrs}$. Cells on the upper surface of the filters were gently removed by wiping with a cotton swab. The cells under the surface of the lower chamber were fixed in $4 \%$ paraformaldehyde, stained with $1 \%$ crystal violet and counted (Ten random $200 \times$ fields per well). The mean number of cells per field of view was calculated.

\section{Western blot analysis}

Total cell proteins were isolated with a total protein extraction kit (Keygen). Concentration of proteins was determined with the BCA protein assay kit (Cowin BioTech, Beijing, China). $30 \mu \mathrm{g}$ of protein was loaded and 
separated in $10 \%$ sodium dodecyl sulfate polyacrylamide gel electrophoresis (SDS-PAGE) gel and transferred to polyvinylidine difluoride membranes (Millipore, Bedford, MA). The antibody against OLFM4 (Lifespan, rabbit anti-human polyclonal, Seattle, USA) was used to analyze protein expression. Proteins were visualized using horseradish peroxidase-conjugated secondary antibody. Signal was detected by enhanced chemoluminescence techniques (Millipore). Detection of GAPDH with specific antibody (Cell Signaling Technology) was used as the loading control.

\section{Quantitative real-time reverse transcription PCR}

Total RNA and miRNA were prepared from cultured cells or paraffin-embedded tissues, respectively, using Trizol reagent (Invitrogen Life Technology) according to the manufacturer's protocol. $1000 \mathrm{ng}$ of total RNA was converted to cDNA with a First Strand cDNA Synthesis Kit (Toyobo, Osaka, Japan). Reactions were conducted in a 20 ul reaction volume in triplicate using FastStart Universal SYBR Master (Rox; Roche, Mannheim, Germany). SYBR PrimeScript miRNA RTPCR Kit (Takara, Japan) was used for real-time PCR reaction of miRNA. Expression fold-change of genes was evaluated using $2^{-\Delta \Delta \mathrm{Ct}}$. PCR primer sequences included OLFM4 sense ACTGTCCGAATTGACATCATGG, antisense TTCTGAGCTTCCACCAAAACTC; ESR1 sense GGTGCCCTACTACCTGGAGAA, antisense GCCATACTTCCCTTGTCATTG. PGR sense ACACCTCCAGTTCTTTGCTGAC, antisense ATTCTTTCATCCGCTGTTCATT; GAPDH sense AGAAGGCTGGGGCTCATTTG, antisense AGGGGCCATCCACAGTCTTC; miR-486-5p sense 5'-GTACTGAGCTGCCCCGAGAAA-3', antisense UnimiR qPCR Primer; U6 sense Universal_RNU6B_Primer, and antisense Uni-miR qPCR Primer.

\section{Statistical analyses}

Statistical analyses were performed using GraphPad Prism 5.0 software. Student's t-test or ANOVA was used for statistical comparisons of quantitative data. The differences of OLFM4, ER $\alpha$ and PR expression among multiple groups were analyzed by a chi-square test, Fisher's exact test, or the Wilcoxon rank sum test. Associations between OLFM4 expression and ER $\alpha$ or PR and between OLFM4 and miR-486-5p were analyzed by Pearson chi-squared test or Spearman rank correlation test. The relationships between OLFM4 expression and clinico-pathological characteristics were analyzed by the chi-squared test. The Kaplan-Meier method was used to calculate the survival curve. A log-rank test was used for univariate survival analysis. A $P<0.05$ was considered statistically significant. When comparing two groups, the Bonferroni method was used to adjust the inspection standard.

\section{ACKNOWLEDGMENTS}

This study was funded by the National Natural Science Foundation of China (No. 81172485 and No. 31070733) and the Ph.D. Program Foundation of Ministry of Education of China (No. 20130171110007).

\section{CONFLICTS OF INTEREST}

The authors confirm that there are no conflicts of interest.

\section{REFERENCES}

1. Zhao YN, Chen GS and Hong SJ. Circulating MicroRNAs in gynecological malignancies: from detection to prediction. Exp Hematol Oncol. 2014; 3:14.

2. Cheng EJ, Kurman RJ, Wang M, Oldt R, Wang BG, Berman DM and Shih Ie M. Molecular genetic analysis of ovarian serous cystadenomas. Lab Invest. 2004; 84:778784.

3. O'Donnell AJ, Macleod KG, Burns DJ, Smyth JF and Langdon SP. Estrogen receptor-alpha mediates gene expression changes and growth response in ovarian cancer cells exposed to estrogen. Endocr Relat Cancer. 2005; 12:851-866.

4. Lindgren P, Backstrom T, Mahlck CG, Ridderheim M and Cajander S. Steroid receptors and hormones in relation to cell proliferation and apoptosis in poorly differentiated epithelial ovarian tumors. Int J Oncol. 2001; 19:31-38.

5. Burges A, Bruning A, Dannenmann C, Blankenstein T, Jeschke U, Shabani N, Friese K and Mylonas I. Prognostic significance of estrogen receptor alpha and beta expression in human serous carcinomas of the ovary. Arch Gynecol Obstet. 2010; 281:511-517.

6. Zhang X, Huang Q, Yang Z, Li Y and Li CY. GW112, a novel antiapoptotic protein that promotes tumor growth. Cancer Res. 2004; 64:2474-2481.

7. Zhang J, Liu WL, Tang DC, Chen L, Wang M, Pack $\mathrm{SD}$, Zhuang $\mathrm{Z}$ and Rodgers GP. Identification and characterization of a novel member of olfactomedin-related protein family, hGC-1, expressed during myeloid lineage development. Gene. 2002; 283:83-93.

8. Rosenbauer F, Wagner K, Zhang P, Knobeloch KP, Iwama A and Tenen DG. pDP4, a novel glycoprotein secreted by mature granulocytes, is regulated by transcription factor PU.1. Blood. 2004; 103:4294-4301.

9. Kulkarni NH, Karavanich CA, Atchley WR and Anholt RR. Characterization and differential expression of a human gene family of olfactomedin-related proteins. Genet Res. 2000; 76:41-50. 
10. Yu L, Wang L and Chen S. Olfactomedin 4, a novel marker for the differentiation and progression of gastrointestinal cancers. Neoplasma. 2011; 58:9-13.

11. Liu W, Zhu J, Cao L and Rodgers GP. Expression of hGC1 is correlated with differentiation of gastric carcinoma. Histopathology. 2007; 51:157-165.

12. Kobayashi D, Koshida S, Moriai R, Tsuji N and Watanabe $\mathrm{N}$. Olfactomedin 4 promotes S-phase transition in proliferation of pancreatic cancer cells. Cancer Sci. 2007; 98:334-340.

13. Koshida S, Kobayashi D, Moriai R, Tsuji N and Watanabe N. Specific overexpression of OLFM4(GW112/HGC-1) mRNA in colon, breast and lung cancer tissues detected using quantitative analysis. Cancer Sci. 2007; 98:315-320.

14. Luo Z, Zhang Q, Zhao Z, Li B, Chen J and Wang Y. OLFM4 is associated with lymph node metastasis and poor prognosis in patients with gastric cancer. J Cancer Res Clin Oncol. 2011; 137:1713-1720.

15. Liu W, Liu Y, Zhu J, Wright E, Ding I and Rodgers GP. Reduced hGC-1 protein expression is associated with malignant progression of colon carcinoma. Clin Cancer Res. 2008; 14:1041-1049.

16. Chen L, Li H, Liu W, Zhu J, Zhao X, Wright E, Cao L, Ding I and Rodgers GP. Olfactomedin 4 suppresses prostate cancer cell growth and metastasis via negative interaction with cathepsin D and SDF-1. Carcinogenesis. 2011; 32:986994.

17. Yu L, He M, Yang Z, Chen G, Li M, Wang L and Chen $\mathrm{S}$. Olfactomedin 4 is a marker for progression of cervical neoplasia. Int J Gynecol Cancer. 2011; 21:367-372.

18. Duan C, Liu X, Liang S, Yang Z, Xia M, Wang L, Chen $\mathrm{S}$ and $\mathrm{Yu}$ L. Oestrogen receptor-mediated expression of Olfactomedin 4 regulates the progression of endometrial adenocarcinoma. J Cell Mol Med. 2014; 18:863-874.

19. Small EM, O'Rourke JR, Moresi V, Sutherland LB, McAnally J, Gerard RD, Richardson JA and Olson EN. Regulation of PI3-kinase/Akt signaling by muscleenriched microRNA-486. Proc Natl Acad Sci U S A. 2010; 107:4218-4223.

20. Oh HK, Tan AL, Das K, Ooi CH, Deng NT, Tan IB, Beillard E, Lee J, Ramnarayanan K, Rha SY, Palanisamy N, Voorhoeve PM and Tan P. Genomic loss of miR-486 regulates tumor progression and the OLFM4 antiapoptotic factor in gastric cancer. Clin Cancer Res. 2011; 17:26572667.

21. Mees ST, Mardin WA, Sielker S, Willscher E, Senninger $\mathrm{N}$, Schleicher C, Colombo-Benkmann M and Haier J. Involvement of CD40 targeting miR-224 and miR-486 on the progression of pancreatic ductal adenocarcinomas. Ann Surg Oncol. 2009; 16:2339-2350.

22. Rask L, Balslev E, Sokilde R, Hogdall E, Flyger H, Eriksen $\mathrm{J}$ and Litman T. Differential expression of miR-139, miR486 and miR-21 in breast cancer patients sub-classified according to lymph node status. Cell Oncol (Dordr). 2014;
$37: 215-227$

23. Wang J, Tian X, Han R, Zhang X, Wang X, Shen H, Xue L, Liu Y, Yan X, Shen J, Mannoor K, Deepak J, Donahue JM, Stass SA, Xing L and Jiang F. Downregulation of miR486-5p contributes to tumor progression and metastasis by targeting protumorigenic ARHGAP5 in lung cancer. Oncogene. 2014; 33:1181-1189.

24. Sun H, Cui C, Xiao F, Wang H, Xu J, Shi X, Yang Y, Zhang Q, Zheng X, Yang X, Wu C and Wang L. MiR-486 regulates metastasis and chemosensitivity in hepatocellular carcinoma by targeting CLDN10 and CITRON. Hepatol Res. 2015; 45:1312-22.

25. Song L, Lin C, Gong H, Wang C, Liu L, Wu J, Tao S, Hu B, Cheng SY, Li M and Li J. miR-486 sustains NFkappaB activity by disrupting multiple NF-kappaB-negative feedback loops. Cell Res. 2013; 23:274-289.

26. Pang W, Tian X, Bai F, Han R, Wang J, Shen H, Zhang X, Liu Y, Yan X, Jiang F and Xing L. Pim-1 kinase is a target of miR-486-5p and eukaryotic translation initiation factor $4 \mathrm{E}$, and plays a critical role in lung cancer. Mol Cancer. 2014; $13: 240$.

27. Borjigin N, Ohno S, Wu W, Tanaka M, Suzuki R, Fujita K, Takanashi M, Oikawa K, Goto T, Motoi T, Kosaka T, Yamamoto K and Kuroda M. TLS-CHOP represses miR486 expression, inducing upregulation of a metastasis regulator PAI-1 in human myxoid liposarcoma. Biochem Biophys Res Commun. 2012; 427:355-360.

28. Xie YL, Yang YJ, Tang C, Sheng HJ, Jiang Y, Han K and Ding LJ. Estrogen combined with progesterone decreases cell proliferation and inhibits the expression of Bcl-2 via microRNA let-7a and miR-34b in ovarian cancer cells. Clin Transl Oncol. 2014; 16:898-905.

29. Li S, Li Y, Wen Z, Kong F, Guan X and Liu W. microRNA-206 overexpression inhibits cellular proliferation and invasion of estrogen receptor alphapositive ovarian cancer cells. Mol Med Rep. 2014; 9:17031708.

30. Lu Z, Zhang Y, Yan X, Chen Y, Tao X, Wang J, Jia N, Lyu T, Ding J, Feng W and Hua K. Estrogen stimulates the invasion of ovarian cancer cells via activation of the PI3K/ AKT pathway and regulation of its downstream targets Ecadherin and alphaactinin4. Mol Med Rep. 2014; 10:24332440.

31. Dassen H, Punyadeera C, Delvoux B, Schulkens I, Marchetti C, Kamps R, Klomp J, Dijcks F, de Goeij A, D'Hooghe T, Kyama C, Ederveen A, Dunselman G, Groothuis $\mathrm{P}$ and Romano A. Olfactomedin-4 regulation by estrogen in the human endometrium requires epidermal growth factor signaling. Am J Pathol. 2010; 177:2495-2508. 